\title{
Thyrotoxic Periodic Paralysis Presenting with Hypokalemia: A Case Report
}

\author{
Zohair Al Aseri* \\ Department of Emergency Medicine and Critical Care, King Saud University, Saudi Arabia
}

*Corresponding author: Zohair Al Aseri, Department of Emergency Medicine and Critical Care, College of Medicine, King Saud University, Saudi Arabia

To Cite This Article: Zohair Al Aseri. Thyrotoxic Periodic Paralysis Presenting with Hypokalemia: A Case Report. 2020 - 9(5). AJBSR.MS.ID.001438. DOI: 10.34297/AJBSR.2020.09.001438.

Received: 㘹 July 08, 2020; Published: 制 July 30, 2020

\begin{abstract}
Background: Thyrotoxic periodic paralysis (TPP), a serious complication of hyperthyroidism, is a common etiology of secondary hypokalemic periodic paralysis (HPP). In this study, we present a case report of a patient who presented to our ED with weakness and hypokalemia and was found to have severe hyperthyroidism.

Case presentation: A 29-year-old Chinese man presented to our Emergency Department complaining of feeling very weak and tired for 8 hours. The weakness was limited to his four limbs. On physical examination, he was conscious and oriented to place, persons, and time. Upper and lower limb exams showed a power of 4/5 with normal sensation and an absence of Babinski signs. The initial blood work was normal, except for mild hypokalemia; therefore, the initial plan was to start him on one liter of IV normal saline mixed with $40 \mathrm{mEq} \mathrm{KCl}$ over 4 hours before repeating the laboratory investigations. The repeated blood work showed a drop in his potassium level to $2.2 \mathrm{mmol} / \mathrm{L}$, indicating the need for further potassium replacement and extensive blood work, including a thyroid function test, which showed severe hyperthyroidism. The patient was admitted to the critical care unit, where his vital signs were monitored continuously, and he received $\mathrm{KCl}$ replacement and began propranolol and carbimazole treatment.
\end{abstract}

Conclusions: TPP should be a differential diagnosis for patients with unexplained hypokalemia and weakness, even in the absence of clinical features of hyperthyroidism.

\section{Background}

Hypokalemic periodic paralysis (HPP) is a severe emergency condition that requires immediate attention, treatment, and admission to the critical care unit; otherwise, it can lead to lifethreatening complications such as arrhythmias and respiratory failure. It is characterized by muscle paralysis and hypokalemia. Thyrotoxic periodic paralysis (TPP) is a serious complication of hyperthyroidism, and it is a common etiology of secondary HPP. In this study, we present a case report of a patient who presented to our ED with weakness and hypokalemia and was found to have severe hyperthyroidism.

\section{Case Presentation}

A 29-year-old Chinese man presented to our Emergency Department complaining of feeling very weak and tired for 8 hours. The weakness was limited to his four limbs, and he had no blurred vision, slurred speech, difficulty in breathing, or chest pain. He denied recently losing weight. He denied palpitations, heat intolerance, chronic diarrhea, recent viral illness, or contact with sick persons and reported that he did not drink alcohol or smoke cigarettes. He denied engaging in physical exertion or consuming a carbohydrate-rich meal before experiencing these symptoms. He had a similar episode 7 years earlier, and the symptoms improved after visiting a local emergency department. He had no recent travel history to epidemic regions. His family history was significant for diabetes mellitus.

On physical examination, he was conscious and oriented to place, persons, and time. He had normal vital signs, including heart rate, blood pressure, respiratory rate, and temperature; his cranial nerve exams were normal. He had no exophthalmos, no lid lag, and no palpable neck masses. His chest, heart, abdominal, and skin 
exams were normal. Upper and lower limb exams showed a power of $4 / 5$ with normal sensation and an absence of Babinski signs.

The initial blood work was normal, except for mild hypokalemia (Table 1); therefore, the initial plan was to start him on one liter of IV normal saline mixed with $40 \mathrm{mEq} \mathrm{KCl}$ over 4 hours before repeating the laboratory investigations, at which point he was to be discharged home if his potassium level had normalized. The repeated blood work showed a drop in his potassium level to $2.2 \mathrm{mmol} / \mathrm{L}$, which indicated the need for further potassium replacement and extensive blood work, including a thyroid function test, which showed severe hyperthyroidism. The patient was referred to critical care and endocrinology for further management of hypokalemia and thyrotoxicosis.

\begin{tabular}{|c|c|c|}
\hline \multicolumn{3}{|l|}{ Table 1: Laboratory investigations. } \\
\hline Laboratory parameter & Results & Normal range \\
\hline Sodium (mmol/L) & 139 & $135-145$ \\
\hline Potassium (mmol/L) & 3.3 & $3.2-5.0$ \\
\hline $\begin{array}{c}\text { Inorganic phosphate } \\
\text { (mmol/L) }\end{array}$ & 1.5 & $0.81-1.45$ \\
\hline Magnesium (mmol/L) & 1.8 & $0.74-1.00$ \\
\hline Corrected calcium (mmol/L) & 2.2 & $2.06-2.44$ \\
\hline Urea (mmol/L) & 4.3 & $2.0-6.7$ \\
\hline Creatinine ( $\mu \mathrm{mol} / \mathrm{L})$ & 50 & $45-84$ \\
\hline Creatine kinase (U/L) & 554 & $30-170$ \\
\hline $\begin{array}{c}\text { Thyroid-stimulating } \\
\text { hormone ( } \mu \mathrm{IU} / \mathrm{mL})\end{array}$ & $0.015(\mathrm{Low})$ & $0.270-4.200$ \\
\hline Free T4 (pmol/L) & $81.6(\mathrm{High})$ & $22-\mathrm{Dec}$ \\
\hline Free T3 (pmol/L) & $35.0(\mathrm{High})$ & $4-8.3$ \\
\hline Chloride $(\mathrm{mmol} / \mathrm{L})$ & 103 & $98-107$ \\
\hline Lactic acid $(\mathrm{mmol} / \mathrm{L})$ & 1.8 & $01-2.0$ \\
\hline
\end{tabular}

The patient was admitted to the critical care unit, where his vital signs were monitored continuously, and he received $\mathrm{KCl}$ replacement and was started on propranolol and carbimazole. His weakness and potassium level improved over the course of his admission to the critical care unit, and he was discharged in excellent condition with follow-up appointment with the Endocrinology Department.

\section{Discussion}

In thyrotoxicosis, $\mathrm{Na} / \mathrm{K}-\mathrm{ATPase}$ overactivity plays a primary role in inducing muscle weakness and hypokalemia through intracellular potassium shifts that are aggravated by stress, catecholamine, and high levels of insulin that are released after consuming a meal rich in carbohydrates [1]. TPP can induce HPP; therefore, all patients with HPP should be investigated for TPP. In a retrospective study by Kalitha et al. [2], a review of 52 patients with HPP found that TPP accounts for approximately 17.3\% of HPP, and there are no differences in demographic, clinical, biochemical and outcome profiles between idiopathic HPP and TPP. The diagnosis of TPP can be challenging because two-thirds of TPP patients have subclinical thyrotoxicosis at the time of presentation. Hegde et al. [3] reported a patient known to have Graves' disease who came to their ED with the clinical profile of HPP without the signs and symptoms of thyrotoxicosis. TTP and hypokalemia can be the initial presentation of thyrotoxicosis and Graves' disease [4-6]. Rebound hyperkalemia is a known complication during the treatment of TTP and HPP. In their review study, of the 52 HPP patients, 8 experienced rebound hyperkalemia; it is crucial to monitor the serum potassium level during treatment to avoid such complications [2]. The serum potassium level can be very low in patients with TPP $[4,7]$.

However, normal potassium levels during paralytic attacks does not exclude the diagnosis of TPP. Several studies reported patients with TTP who presented at the ED with normokalemia and muscle paralysis. Serial monitoring of potassium levels is indicated to enable the observation of any progressive drop in serum potassium levels following admission [8,9]. In a study of 23 HPP patients by Jandhyala et al. [10], 13 (57\%) had primary HPP, and 10 (43\%) had secondary HPP. Men were predominantly affected [10]. Diarrhea, fever, strenuous activity, a meal rich in carbohydrates, and febrile illness are common precipitating factors [11-14]. In another study of 31 patients by Rao et al. [15], the secondary causes of HPP were analyzed; 13 patients were diagnosed with renal tubular acidosis, and two were diagnosed with TTP. Of the 31 patients, 19 were men [15]. Although hyperthyroidism is more common in females, TPP with secondary HPP occurs relatively more frequently in males $[10,15,16]$.

\section{Conclusions}

TPP should be one of the differential diagnoses of patients with unexplained hypokalemia and weakness, even in the absence of clinical features of hyperthyroidism.

\section{Declarations}

Ethics approval and consent to participate. This case report is approved by the IRB of the College of Medicine at King Saud University.

\section{Consent for Publication}

Written informed consent was obtained from the patient for publication of this case report.

\section{Availability of data and materials}

Not applicable

\section{Competing interests}

The author declares that he has no competing interests.

\section{Funding}

Not applicable. 


\section{Author contributions}

ZA participated in the diagnosis and treatment of the patient, provided follow-up, acquired clinical data, drafted and reviewed the manuscript, conducted investigations, reviewed the literature, and polished the manuscript for final publication.

\section{Acknowledgements}

Not applicable.

\section{Reference}

1. Kasper D, Fauci A, Hauser S, Longo D, Jameson L, et al. (2015) Harrison's principles of internal medicine. McGraw Hill, New York, USA.

2. Kalita J, Goyal G, Bhoi SK, Chandra S, Misra UK, et al. (2012) Comparative study of thyrotoxic periodic paralysis from idiopathic hypokalemic periodic paralysis: an experience from India. Ann Indian Acad Neurol 15(3): 186-190.

3. Hegde S, Shaikh MA, Gummadi T (2016) Hypokalaemic periodic paralysis in a patient with subclinical hyperthyroidism: a rare case. J Clin Diagn Res 10(1): OD14-OD15.

4. Meseeha M, Parsamehr B, Kissell K, Attia M (2017) Thyrotoxic periodic paralysis: a case study and review of the literature. J Community Hosp Intern Med Perspect 7(2):103-106.

5. Chang C C, Cheng C J, Sung C C, Chiueh T S, Lee C H, et al. (2013) A 10year analysis of thyrotoxic periodic paralysis in 135 patients: focus on symptomatology and precipitants. Eur J Endocrinol 169(5): 529-536.

6. Alqahtani SF, Aleithan MM (2017) Thyrotoxic periodic paralysis as an initial presentation of Graves' disease in a Saudi patient. BMJ Case Rep 2017.
7. Maurya PK, Kalita J, Misra UK (2010) Spectrum of hypokalaemic periodic paralysis in a tertiary care centre in India. Postgrad Med J 86(1022): 692-695.

8. Chakrabarti S (2015) Normokalemic thyrotoxic periodic paralysis with preserved reflexes- a unique case report. J Clin Diagn Res 9(2): OD05OD06.

9. Valizadeh N, Zarrin S (2013) A 32-year-old man with normokalemic thyrotoxic periodic paralysis. Malays J Med Sci 20(1): 81-83.

10. Jandhyala SN, Madireddi J, Belle J, Rau NR, Shetty R, et al. (2015) Hypokalaemic periodic paralysis-a prospective study of the underlying etiologies. J Clin Diagn Res 9(9): 0C17-9.

11. Klubo Gwiezdzinska J, Wartofsky L (2012) Thyroid emergencies. Med Clin North Am 96(2): 385-403.

12. Vijayakumar A, Ashwath G, Thimmappa D (2014) Thyrotoxic periodic paralysis: clinical challenges. J Thyroid Res 2014: 649502.

13. Kumar MR, Bharath R, Rammohan P, Agrawal A (2014) Clinical profile in hypokalemic periodic paralysis cases. Eur J Gen Med 11(1): 6-9.

14. Al Moteri BLG, Aslam M (2017) Thyrotoxic periodic paralysis: a case report. Int J Health Sci 11(1): 1-2.

15. Rao N, John M, Thomas N, Rajaratnam S, Seshadri MS, et al. (2006) Aetiological, clinical and metabolic profile of hypokalaemic periodic paralysis in adults: a single-centre experience. Natl Med J India 19(5): 246-249.

16. Hsieh MJ, Lyu RK, Chang WN, Chang KH, Chen CM, et al. (2008) Hypokalemic thyrotoxic periodic paralysis: clinical characteristics and predictors of recurrent paralytic attacks. Eur J Neurol 15(6): 559-564. 